submitted to Phys. Rev. E

\title{
Scaling of waves in the Bak-Tang-Wiesenfeld sandpile model
}

\author{
D. V. Ktitarev ${ }^{1,2 *}$, S. Lübeck ${ }^{2}$, P. Grassberger ${ }^{1}$ and V. B. Priezzhev ${ }^{3}$ \\ ${ }^{1}$ John von Neumann Institut für Computing, Forschungszentrum Jülich, 52425 Jülich, Germany \\ ${ }^{2}$ Theoretische Physik, Gerhard-Mercator-Universität Duisburg, 47048 Duisburg, Germany \\ ${ }^{3}$ Laboratory of Theoretical Physics, Joint Institute for Nuclear Research, Dubna, 141980 Russia
}

(June 30, 1999)

\begin{abstract}
We study probability distributions of waves of topplings in the Bak-Tang-Wiesenfeld model on hypercubic lattices for dimensions $D \geq 2$. Waves represent relaxation processes which do not contain multiple toppling events. We investigate bulk and boundary waves by means of their correspondence to spanning trees, and by extensive numerical simulations. While the scaling behavior of avalanches is complex and usually not governed by simple scaling laws, we show that the probability distributions for waves display clear power law asymptotic behavior in perfect agreement with the analytical predictions. Critical exponents are obtained for the distributions of radius, area, and duration, of bulk and boundary waves. Relations between them and fractal dimensions of waves are derived. We confirm that the upper critical dimension $D_{\mathrm{u}}$ of the model is 4 , and calculate logarithmic corrections to the scaling behavior of waves in $D=4$. In addition we present analytical estimates for bulk avalanches in dimensions $D \geq 4$ and simulation data for avalanches in $D \leq 3$. For $D=2$ they seem not easy to interpret.
\end{abstract}

\section{INTRODUCTION}

The sandpile model was introduced by Bak, Tang, and Wiesenfeld (BTW) [1] as a simple example of a slowly driven dissipative system exhibiting self-organized criticality (SOC). Although today many systems with SOC are known, it is considered as the prototype of such models, and there is a huge literature devoted to it. Its theoretical understanding is crucially related to Dhar's discovery of its Abelian structure [2] which allows exact calculation of many of its properties [3,4]. However, a complete analytical determination of the scaling behavior of avalanches is still lacking. Several approximation schemes, including a random walk approach |5], diffusionlike analogy [6], renormalization group [7],8] and a graph theory method [9] were proposed, but led to different results. In addition, computer simulations - which first had suggested simple scaling behavior together with standard finite-size scaling (FSS) - provide increasing evidence that the avalanche statistics is much more complicated. While most recent authors agree upon a breakdown of FFS, the detailed interpretation of their data is highly controversial among different groups [10 13].

The standard FSS ansatz implies an asymptotic form

$$
P_{a}(a, L) \sim a^{-\tau_{a}} p\left(a / L^{\nu_{a}}\right)
$$

for the distribution of the number $a$ of toppled sites in an avalanche (in other words, its "area"), where $L$ is the size of the lattice, $p(z)$ is a universal function, and $\tau_{a}$ and $\nu_{a}$ are critical exponents. This ansatz implies simple scaling of all moments of $a,\left\langle a^{n}\right\rangle \sim L^{\sigma_{n}}$ with $\sigma_{n}=\sigma_{0}+n \nu_{a}$. Similar ansatzes should, according to this view, hold for the number of topplings $s$ (which differs from $a$ because sites can topple more than once in an avalanche) and for the radius and duration of avalanches. But recent investigations 10 12. show that the two-dimensional BTW model may be characterized by a multifractal behavior where different moments of $a$ are governed by exponents $\sigma_{n}$ which are not linear in $n$ and are indeed not related to each other for different $n$. Different reasons for this have been proposed in [10,11] and in [12]. Notice that multifractality of avalanches can be proven for certain variants of the 1- $d$ sandpile model [14].

Deviations from pure power laws had been seen already in early simulations, but were usually interpreted as finite size effects due to avalanches which touch the boundary of the lattice. To illustrate that this is most likely not true, and that there is a real problem with simple scaling, we show in Fig. 1 1 the ratio $\mathcal{P}_{s}(x, L) / \mathcal{P}_{a}(x, L)$ of the integrated distributions $\mathcal{P}_{s}(x, L)=\int_{x}^{\infty} d x^{\prime} P_{s}\left(x^{\prime}, L\right)$ for $D=2$ and for different values of $L$. In these simulations, cylindrical boundary conditions were used (open at $y= \pm L / 2$ and periodic at $x=0, L$ ), and data were collected only for avalanches starting at $y=0$. In this way we hope to have minimized boundary effects. Also, since we do not make separate fits to $P_{s}(x, L)$ and $P_{a}(x, L)$, we have none of the uncertainties inherent in such fits. Due to Eq. (11) we would expect this ratio to scale as $x^{\tau_{a}-\tau_{s}}$ for $x \ll \min \left\{L^{\nu_{a}}, L^{\nu_{s}}\right\} \approx L^{2}$. According to analytical prediction 15] and recent large scale simulations 12, 13, 16, the difference $\tau_{a}-\tau_{s}$ should be in the range 0.024 to 0.08 . The behavior seen in Fig. 1 is rather different. Although the curves for different large $L$ perfectly superimpose in a wide range, they are in this range not straight at all (as expected for a power law), and their average slope in this universal range is much smaller. Very small differences $\tau_{a}-\tau_{s}$ have been seen in several simulations using small lattices 17,18. But it still disagrees with our data showing a lack of scaling even for avalanches which do not reach the boundary of the lattice. Most other variables show similar deviations from pure power laws in $D=2$ when scrutinized closely.

In principle, one can expect that these deviations of scaling can be explained by assuming that the avalanche boundary advances like a pinned surface in a random medium. Unfortunately, this interpretation seems untenable. As shown in [19] (see also [20]), avalanches proceed in distinct waves of topplings. In each wave, any site top- 


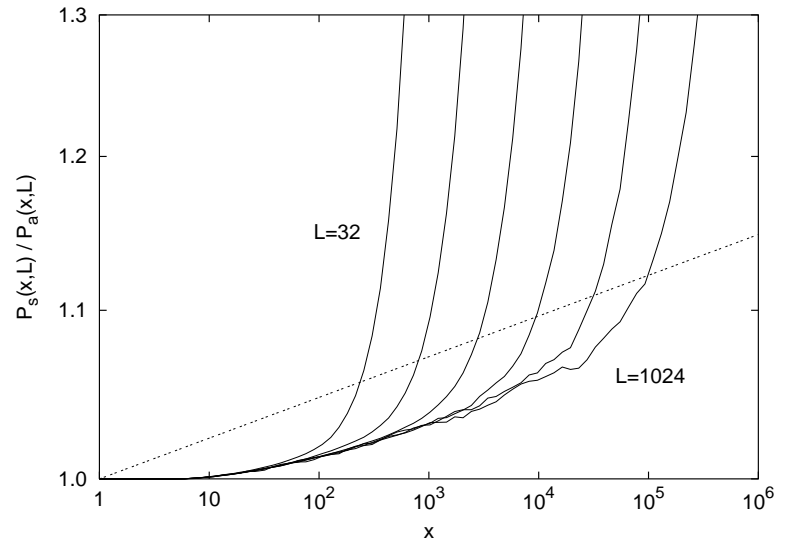

FIG. 1. Ratio $\mathcal{P}_{s}(x, L) / \mathcal{P}_{a}(x, L)$ of the integrated $s$ - and $a$-distributions for two-dimensional sandpiles. According to the generally accepted FSS ansatz this should be a power law with exponent $\tau_{a}-\tau_{s}$ in the range 0.024 to 0.08 in the region where it is independent of $L$. The dashed line is $x^{0.01}$.

ples at most once. In the original version of the model waves overlap in time, but they can be disentangled by a simple trick [19] so that at any time only a single wave propagates. Therefore, if at all, the arguments associated with pinning effects should not apply to boundaries of avalanches but to the propagation of wave boundaries, and they would suggest that waves show complex behavior (notice that boundaries of successive waves are not simply related to each other [21).

But waves do behave simply, and do show simple scaling behavior. This is indeed the main message of the present paper. Our results extend analytical results derived in 19,22, 23, and large scale simulations made in [24,21. While the behavior of avalanches is complex and badly understood when typical avalanches are composed of many waves (which is the case for $D=2$ and, to a much less degree, for $D=3$ ), the behavior of single waves is simple and well understood.

In particular, we show in the present paper that FSS works for waves of topplings. Since boundary avalanches (i.e. avalanches which start from an unstable boundary site) always consist of single waves [19], it applies also to them. Using the spanning tree representation of waves, the equivalence between spanning trees and loop-erased random walks, and rigorous estimations for the latter, we determine the critical exponents of their probability distributions for all dimensions $D \geq 2$. We also use the wave statistics to confirm recent numerical [25] and analytical [5,26] predictions for the upper critical dimension of the BTW sandpile model. The upper and lower bounds for logarithmic corrections to scaling for four-dimensional waves are determined analytically and confirmed numerically.

We discuss the possibility for investigation of avalanche distributions of the BTW model using the results obtained for waves. One of the key characteristics in this study is the average number $\langle n\rangle_{a}$ of waves in avalanches of a given area $a$. Our simulations show that in $D=2$ this number grows, most probably, not slower than a power law with the exponent 0.17 (the first conjecture of 15] was $1 / 6$, and an analytical prediction of [9] is $1 / 4)$. This means that multiple topplings substantially change the scaling behavior of avalanches in comparison to waves, which was indicated in many previous studies of the two-dimensional BTW model.

In $D=3$ the fraction of avalanches containing more than one wave is much less than in two dimensions. All accurate numerical estimations of the exponent $\tau_{a}$ lead to $\tau_{a}=1.33$ which coincides with the exact exponent $4 / 3$ for the wave distribution, which could mean that the averaged number of waves in an avalanche in $D=3$ grows not faster than logarithmically. On the other hand, considering the numerical estimation of this number we cannot exclude its slow polynomial growth with the avalanche size. Then, the scaling behavior of avalanches could be corrected for the multiple topplings in large events, similar to the case $D=2$.

Finally, for $D \geq 4$ the upper logarithmic bound for the averaged number of waves in an avalanche [26] implies that the distributions of avalanches obey asymptotic behavior with the same exponents as for waves.

This paper is organized as follows. In section [1] we remind basic definitions of the BTW model. Section III is devoted to detailed explanation of the construction of waves and their spanning tree representation. In section IV we derive the critical exponents of wave distributions. In section $\mathrm{V}$ we discuss analytical results for the dynamical exponent and fractal dimension of waves. Results of computer simulations are presented in section $\mathrm{VI}$.

\section{THE BTW MODEL}

We consider the $D$-dimensional BTW model on a hypercubic lattice of linear size $L$ in which integer variables $z_{i} \geq 0$ represent local energies. One perturbs the system by adding particles at randomly chosen sites according to

$$
z_{i} \mapsto z_{i}+1
$$

A site is called unstable if the corresponding energy $z_{i}$ exceeds the critical value $2 D$. An unstable site relaxes, its energy is decreased by $2 D$ and the energy of the $2 D$ nearest neighbors $(\mathrm{nn})$ is increased by one:

$$
\begin{gathered}
z_{i} \rightarrow z_{i}-2 D \\
z_{\mathrm{nn}} \rightarrow z_{\mathrm{nn}}+1 .
\end{gathered}
$$

In this way, the neighboring sites may be activated and an avalanche of relaxations may proceed. If a boundary site topples, one or more particles leave the system. The avalanche of relaxations stops when all sites are stable again.

One can introduce in a natural way different kinds of sub-avalanches, e.g. clusters of sites toppled not less than a given number of times [20] or waves of topplings [19]. A relaxation event (an avalanche or sub-avalanche) is characterized by its size $s$ (total number of topplings), area 
$a$ (number of distinct toppled sites), duration $t$ (number of parallel update steps until stable configuration is reached), and its radius $r$ (e.g. the radius of gyration or the maximal distance between the origin and a toppled site). The basic hypothesis of Bak et al. [1] claimed that in the self-organized critical state the probability distributions of values $s, a, t, r$ exhibit power-law behavior for intermediate values of $x$,

$$
P_{x}(x) \sim x^{-\tau_{x}}
$$

with $x \in\{s, a, t, r\}$.

As we have seen, this hypothesis might not be true for complete avalanches, but as we shall see it does hold for waves. Scaling relations for the exponents $\tau_{s}, \tau_{a}, \tau_{t}$, and $\tau_{r}$ can be obtained if one assumes that size, area, duration and radius of "typical" events scale as powers of each other, for instance

$$
t \sim r^{\gamma_{t r}}
$$

Then the transformation law of probability distributions $P_{t}(t) \mathrm{d} t=P_{r}(r) \mathrm{d} r$ leads to the scaling relation

$$
\gamma_{t r}=\frac{\tau_{r}-1}{\tau_{t}-1} .
$$

Again we should warn that there is a crucial assumption underlying these relations, namely that conditional distributions $P_{x}(x \mid y)$ are narrow, and therefore Eq. (6) holds with small deviations for most events. It was proposed in 12 that this might not be justified in $D=2$, and this is indeed the main source of problems of this approach. Let us ignore this for the moment and proceed nevertheless.

The scaling exponents $\gamma_{x x^{\prime}}$ are important for the description of the extent of avalanches and their propagation. For instance the exponent $\gamma_{s a}$ indicates if multiple toppling events are relevant $\left(\gamma_{s a}>1\right)$ or irrelevant $\left(\gamma_{s a}=1\right)$. The exponent $\gamma_{a r}$ relating the avalanche area to its radius $r$ equals the fractal dimension $D_{f}$ of the avalanche. Finally, the exponent $\gamma_{t r}$ is usually identified with the dynamical exponent $z$.

If Eqs. (57) are applied to waves, one has of course $\gamma_{s a}=1$, but $\gamma_{a r}$ and $\gamma_{t r}$ are non-trivial. Our main result states that Eqs. (57) do indeed apply to waves, together with the FSS ansatz Eq. (1).

\section{WAVES OF TOPPLINGS AND THEIR SPANNING TREE REPRESENTATION}

Dhar proved [2] that all stable configurations can be classified as either transient or recurrent. The former can occur only during an initial transient period, but are irrelevant for the infinite time dynamics. He also formulated the so-called "burning algorithm" which, on the one hand, allows one to distinguish the recurrent states among all stable configurations, and, on the other hand, can be used for constructing a spanning tree representation of any given recurrent state. According to this algorithm, which also proceeds in discrete time steps, any site $i$ is "burnt" at time $t$ if its energy $z_{i}$ is larger than the number of its unburnt nearest neighbors at time $t-1$. In a stable configuration, only some of boundary sites can satisfy this condition at the first step, they can be interpreted as origins of "fire". Then the "fire" propagates if new sites become burnable at the second step. In this way, we burn the sites step by step, until no more sites can be burnt. If all sites of the lattice are burnt, the initial configuration of energies is recurrent, otherwise it it transient.

In order to obtain the spanning tree representation of recurrent configurations 15 , each burnt site $i$ is connected by a bond to one of the sites which had "set it afire", i.e. which had caused its burning by burning itself. If there is more than one such site, one uses an arbitrary but definite set of rules where to place this bond. In addition, one introduces a new site $\square$ ("sink") and connects it to all boundary sites. On these connections, bonds are placed to those sites which burn at $t=1$. Then we can imagine the entire process to start by burning the site $\square$ at time $t=0$, and generating a rooted tree with root at $\square$. If the state is recurrent, this tree spans the entire lattice.

Majumdar and Dhar [15] also noticed that the condition for "toppling" of a site is essentially the same as the condition for "burning": At each step, the site $i$ topples if its energy $z_{i}$ is larger than the number of those of its nearest neighbors which had not toppled in the step before. Therefore, the burning of a recurrent state is equivalent to a toppling process initiated "from the boundary". It implies that if we add one particle to every boundary site (two particles on each corner, etc.), each site will topple exactly once during the ensuing avalanche.

The burning algorithm gives a one-to-one correspondence between recurrent states and spanning trees. This allows one to calculate the total number of recurrent configurations, the energy probabilities and the energyenergy correlation functions [2, 27].

The spanning tree representation can be constructed also for a certain class of unstable configurations appearing during an avalanche. It was shown in 19 that avalanches in the BTW model can be decomposed into so called 'waves of topplings'. According to this construction, an avalanche is considered as a superposition of successive sub-avalanches. After perturbing the system at a given lattice site $i$, one allows it to relax, but prevents the site $i$ temporarily from toppling a second time. After this first 'wave' all sites are again stable except, possibly, the site $i$. If $i$ is unstable, a second wave is initiated by toppling it again. But a possible third toppling is again delayed until this wave is finished, and when it finally occurs, it triggers the third wave. The procedure is repeated until the site $i$ is stable. Note that if the site $i$ is on the boundary, the avalanche stops after first relaxation and consists of only one wave. More generally, if the distance of $i$ to the boundary is $d$, then any avalanche starting at $i$ can have at most $d+1$ waves.

To obtain the tree representation of a configuration following a wave which had started at site $i$, we introduce an auxiliary BTW model on a new lattice. In this lattice 


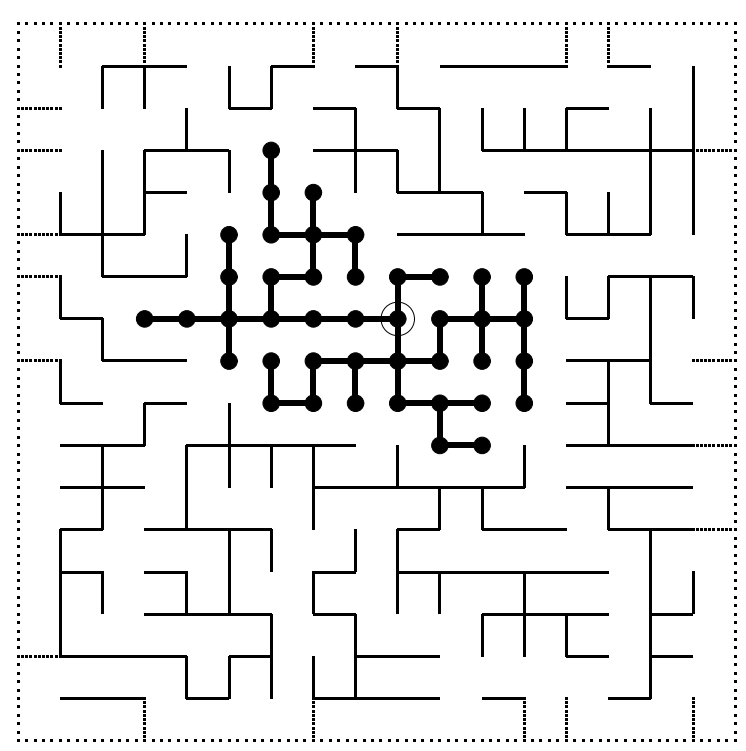

FIG. 2. Spanning 2-tree representation of a wave. Toppled sites are marked by heavy dots. The origin of the wave is marked by a circle. The dotted lines indicate the boundary of the system. The latter is considered as a single additional site $\square$, so that all non-toppled sites form a single connected tree.

we connect $i$ to the sink $\square$. Since the site $i$ in the new model is on the boundary, each avalanche starting at this site will consist of a single wave. Each avalanche in the auxiliary model corresponds indeed to a wave of some avalanche in the original model. Applying the burning algorithm, we can construct a spanning tree on the auxiliary lattice representing the recurrent state of the new model. During this burning process, some branches of the fire will be independent of site $i$, but one branch will first pass from $\square$ to $i$ and then propagate further. It is this latter branch which coincides with the last wave of topplings in the original BTW model.

Removing the bond between $i$ and $\square$ we obtain two trees, one having the root at $i$ and the second at the sink $\square$. The first tree represents the wave and the second one corresponds to the sites not toppled in this wave. The tree with the root $\square$ contains information about the configuration of stable sites not affected by the wave. We will call this union of two trees which cover the entire lattice, a spanning two-component tree (or, simply, a spanning 2 -tree). According to this tree representation, waves with different configurations of untoppled sites are counted as different. An example of a spanning 2-tree is shown in Fig. 2.

The rigorous proof of the above construction is given in 19. A similar decomposition of avalanches into "inverse avalanches" was proposed by Dhar and Manna [22].

An important fact concerning the wave statistics should be noted. Since all recurrent states of the auxiliary BTW model have equal probability of occurrence [2], all waves in the original BTW model are also equally likely.

\section{CRITICAL EXPONENTS AND GREEN FUNCTIONS}

Using the graph representation of waves, we can express their probability distribution by the lattice Green function. Consider avalanches initiated by adding a particle at the site $i$ and spanning 2-trees with roots at $i$ and $\square$ representing waves of these avalanches. It was proven in [19] that the Green function $G_{i j}$ is related to the number $N_{(\square)(i j)}$ of spanning 2 -trees where the site $j$ is in the same component as $i$ :

$$
G_{i j}=\frac{N_{(\square)(i j)}}{N_{(\square)}}
$$

where $N_{(\square)}$ is the total number of spanning trees with the root at the site $\square$.

Consider avalanches initiated by adding a particle at the site $i$. Since every recurrent state together with the perturbed site $i$ completely defines the relaxation process, the number of different possible avalanches $N_{(i)}^{(a)}$ started at fixed point $i$ equals to the number of recurrent states (or the number of spanning trees $N_{(\square)}$ ). The number $N_{(i j)}^{(w)}$ of different waves started at the site $i$ and covering the site $j$ corresponds to the number $N_{(\square)(i j)}$ of spanning 2 -trees having sites $i, j$ on one of its components. We can therefore rewrite Eq. (8) as

$$
G_{i j}=\frac{N_{(i j)}^{(w)}}{N_{(i)}^{(a)}} .
$$

Eq. (9) is another formulation of the known result of Dhar [2] that the expected number of topplings at site $j$ due to adding a particle at the site $i$ is given by the Green function $G_{i j}$.

Due to uniformness of the wave statistics mentioned at the end of section III, the probability that a wave $W(i)$ starting at the site $i$ covers the site $j$ is equal to the fraction of waves having this property:

$$
P(j \in W(i))=\frac{N_{(i j)}^{(w)}}{N_{(i)}^{(w)}},
$$

where $N_{(i)}^{(w)}$ denotes the total number of waves starting at $i$.

Combining Eqs. (9) and (10), we write

$$
P(j \in W(i))=\frac{N_{(i j)}^{(w)}}{N_{(i)}^{(a)}} \frac{N_{(i)}^{(a)}}{N_{(i)}^{(w)}}=\frac{G_{i j}}{G_{i i}}=\frac{G(r)}{G(0)} .
$$

where we use the notation $G(r)$ for the Green function $G_{i j}$ if the points $i$ and $j$ are separated by the distance $r$.

On the other hand, this probability can be represented as

$$
P(j \in W(i))=\int_{r}^{\infty} P^{(w)}(R) \rho_{R}(r) d R,
$$

where $P^{(w)}(R)$ is the probability that the linear extent of a wave is $R$, and $\rho_{R}(r)$ denotes the density of sites 
covered by such a wave. The density $\rho_{R}(r)$ tends to 1 for large $R$ if waves are compact and isotropic, and is a function of $r$ if waves are fractal. Asymptotically for $R \gg r$, it varies as

$$
\rho_{R}(r) \approx \rho(r) \sim r^{d_{f}-D}
$$

where $d_{f}$ is the fractal dimension of waves and $D$ is the Euclidean dimension of the lattice.

Suppose that the probability distribution of the wave radius $r$ has a power law asymptotics similar to that for avalanches [Eq. (5)],

$$
P_{r}^{(w)}(r) \sim r^{-\tau_{r}^{(w)}}
$$

Then, the probability distribution $P_{r}^{(w)}\left(r^{\prime}>r\right)$ scales with the exponent $\tau_{r}^{(w)}-1$. Using Eq. (13) we get the asymptotic behavior of the probability in lhs of Eq. (12)

$$
P(j \in W(i)) \sim r^{-\tau_{r}^{(w)}+1+d_{f}-D} .
$$

The asymptotics of the bulk Green function (see for instance [28]) are given by

$$
G(r) \sim \begin{cases}\ln r & \text { for } D=2 \\ r^{2-D} & \text { for } D>2\end{cases}
$$

which reveals that the radius exponent $\tau_{r}^{(w)}$ for waves is

$$
\tau_{r}^{(w)}=d_{f}-1
$$

Using Eq. (7) we can derive the exponents of the wave area

$$
\tau_{a}^{(w)}=2-\frac{2}{d_{f}}
$$

and duration

$$
\tau_{t}^{(w)}=1+\frac{d_{f}-2}{z}
$$

respectively.

For avalanches started at a distance $b$ from the boundary, we need the boundary Green functions which can be calculated by the method of images:

$$
G(\mathbf{r}) \sim \begin{cases}\ln |\mathbf{r}+\mathbf{b}|-\ln |\mathbf{r}-\mathbf{b}| & \text { for } D=2 \\ |\mathbf{r}-\mathbf{b}|^{2-D}-|\mathbf{r}+\mathbf{b}|^{2-D} & \text { for } D>2\end{cases}
$$

where $\mathbf{b}$ is the vector perpendicular to the boundary. On any "equipotential" surface $G(\mathbf{r})=$ const characterized by a length scale $\xi$ and a volume $a \sim \xi^{D}$, this boundary Green function scales as

$$
G \sim b \xi^{1-D}
$$

If we now replace Eq. (13) (which is appropriate only for isotropic cases) by its generalization $\rho \sim \xi^{d_{f}-D}$, we arrive at the exponents for waves starting near the boundary:

$$
\begin{gathered}
\tau_{r}^{\text {(boundary })}=d_{f}, \\
\tau_{a}^{\text {(boundary })}=2-\frac{1}{d_{f}}, \\
\tau_{t}^{\text {(boundary })}=1+\frac{d_{f}-1}{z}
\end{gathered}
$$

(here and in the following we use superscript $(w)$ for bulk waves and (boundary) for boundary waves, and use symbols without superscripts for avalanches). We see that both the bulk and boundary wave exponents are determined by the scaling exponents $d_{f}$ and $z$. The dynamical exponent $z$ can be related to the fractal dimension of the "chemical path" on a spanning tree [15] which, in turn, is equivalent to the dimension of the loop-erased random walk (LERW) [29]. As to the fractal dimension of waves $d_{f}$, it was proven for all dimensions that a set of untoppled sites, which are completely surrounded by toppled sites, corresponds to a forbidden subconfiguration [17,20]. However, this fact does not prevent waves from being fractal. They still could display either nonfractal or fractal behavior depending on the dimensionality $D$. In the next section, we will show that $d_{f}$ is also closely related to properties of LERW, more precisely to the intersection probability between a LERW and a simple random walk.

\section{LOOP ERASED RANDOM WALKS, DYNAMICAL EXPONENT AND FRACTAL DIMENSION OF WAVES}

In this section, we derive analytical estimates for critical exponents of waves using their spanning tree representation and equivalence between a chemical path on a spanning tree and LERW.

Consider an unrestricted random walk on a hypercubic lattice. The LERW introduced by Lawler [30] is obtained from the simple random walk by deleting all loops along the path. The chemical path between two sites of a tree is the unique path along the tree edges connecting these sites. Majumdar 29] has shown that the chemical path on a spanning tree is statistically equivalent to the LERW, i.e., the average distance $r$ between the starting point and the position after $l$ steps scales as $r \sim l^{\nu}$ with the same exponent $\nu$ for both of them.

In $D=2$, the exponent $\nu=4 / 5$ is known exactly from conformal field theory 31]. In $D=3$, numerical estimates yield $\nu \approx 0.61632$.33. In $D=4$ which is the upper critical dimension for the LERW, $\nu=1 / 2$ and the simple scaling law has logarithmic corrections [34]. For $D>4$, the scaling behavior of the LERW and chemical paths is given by the trivial value $\nu=1 / 2$, as the effects of self-intersections become negligible above the upper critical dimension.

Returning to the BTW model, we notice that sites which topple at a given step of wave propagation coincide with sites deleted at the same step of the burning process, if it is started at the origin of the wave. Since 


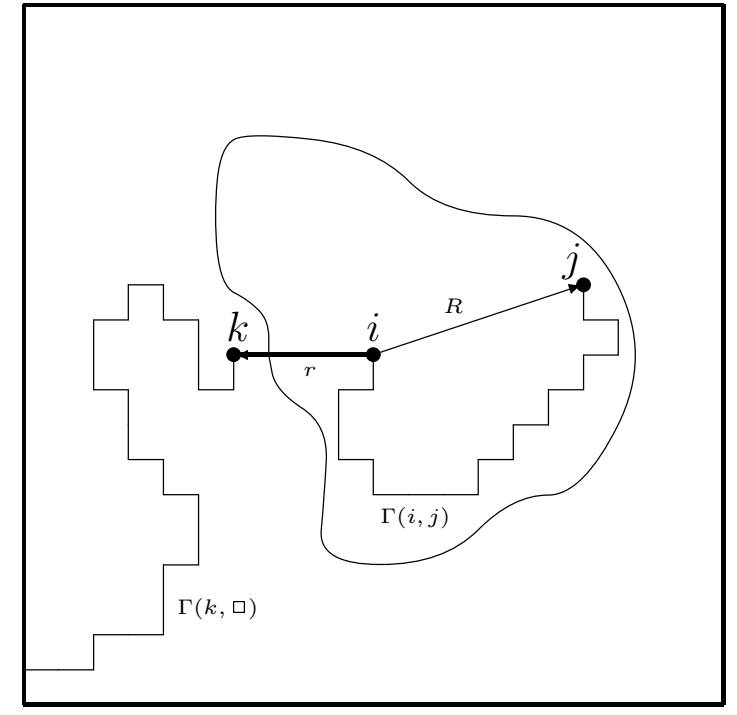

FIG. 3. A sketch of a wave initiated at the site $i$ and containing the site $j$. The chemical path between the two sites on the tree representing the wave is $\Gamma(i, j)$. The second path $\Gamma(k, \square)$ corresponds to a random walk which starts at the site $k$ and escapes the path $\Gamma(i, j)$ until it is trapped at the sink.

the burning process generates a tree, there exist a unique path from the root $i$ of the tree (the site where the wave is initiated) to one of the last toppled sites $i_{f}$ of the wave. The number of update steps is given by the number of edges in this path. Thus, the duration $t$ of the wave equals to $l$, the length of the chemical path from $i$ to $i_{f}$ on the tree and, therefore, the dynamical exponent $\gamma_{t r}$ of waves is given by $z=1 / \nu$.

In order to find the fractal dimension of waves $d_{f}$, we use the proposition proved in 26]. For this we take a site $k$ at distance $r=|k-i|$ from $i$ and a site $j$ at distance $R=|j-i|>r$, together with some paths $\Gamma(i, j)$ connecting $i$ with $j$ and $\Gamma(k, \square)$ connecting $k$ with the sink $\square$ (see Fig. 3). Then the density of sites at distance $r$ from $i$, in waves of radius $R>r$ starting at site $i$, is given by [26]:

$$
\rho_{R}(r)=\overline{P_{\text {int }}(k \square \mid i j)},
$$

where $\overline{P_{\text {int }}(k \square \mid i j)}$ is the probability that $\Gamma(k, \square)$ intersects the path $\Gamma(i, j)$, averaged over all $j$, all paths from $i$ to $j$, all $k$, and all $\Gamma(k, \square)$.

Using the known estimations of the intersection probabilities [34] we can obtain from Eq. (25) the following upper bounds. For $D>4$, we have

$$
\rho(r)=\lim _{R \rightarrow \infty} \rho_{R}(r) \leq C_{1} r^{4-D},
$$

From Eq. (13), we can see that the fractal dimension of waves $d_{f} \leq 4$ for all $D \geq 4$.

For $D=4$, the upper bound reads

$$
\rho_{R}(r) \leq C_{2} \frac{\ln (1+\alpha)}{\ln r}, \quad \alpha=\frac{R^{2}}{r^{2}}
$$

while a lower bound was obtained in [26]:

$$
\rho_{R}(r) \geq 1-C_{3} \frac{(\ln r)^{1 / 2}}{(\ln R)^{1 / 3}}
$$

We can see that $\rho_{R}(r)$ approaches 1 when $R \rightarrow \infty, r$ fixed. The only fractal dimension which is consistent with both upper and lower bounds Eqs. (27,28) is 4, but there are logarithmic corrections.

For $D<4$ the lower bound Eq. (28) becomes stronger because of increasing intersection probability $\overline{P_{\text {int }}(k \square \mid i j)}$. Thus, we conclude that the fractal dimension of waves is

$$
d_{f}= \begin{cases}D & \text { for } D \leq 4 \\ 4 & \text { for } D>4\end{cases}
$$

which means that the upper critical dimension for waves is 4 .

Therefore we can calculate from Eqs. (17, 19, 22,24) the exact values of all exponents for bulk and boundary waves for all dimensions $D \geq 2$, with a single exception. This exception is the exponent of the duration distribution in $D=3$, for which we need the value of $\nu_{\mathrm{LERW}}(D=3)$ which is not known exactly.

\section{COMPARISON WITH NUMERICAL SIMULATIONS}

\section{A. $\mathrm{D}=2$}

In this subsection we present the results of numerical simulations of bulk and boundary waves in $D=2$. For these the standard FSS works well. For any of the observables $x=a, t$, and $r$ it can be written as

$$
P_{x}(x, L)=L^{-\beta_{x}} g_{x}\left(x L^{-\nu_{x}}\right),
$$

with $\beta_{x}=\tau_{x} \nu_{x}$ [35].

The functions $g_{x}(z)$ should be universal (i.e., does not depend on the type of lattice). But for large values of $z$ they do depend on the type of boundary conditions (open on all four sides or cylindrical, i.e. open in one direction and periodic in the other) and on the aspect ratio (square or rectangle with sides $L_{1} \neq L_{2}$ ). We verified that the exponents were independent of boundary conditions and aspect ratios. We verified also that all results were unchanged if we threw in the sand grains with non-uniform density, provided this density was everywhere non-zero. The latter was very useful since it allowed us to obtain much improved statistics from either the boundary or the central region.

Since $d_{f}=2$ for $D=2$, one has $\nu_{a}=d_{f}=2$ and $\nu_{r}=1$, and therefore $\nu_{x}=\gamma_{x r}$ [36]. The results of the previous section, together with $\nu_{t}=z=1 / \nu_{\mathrm{LERW}}=5 / 4$, give

$$
\begin{aligned}
& \beta_{a}^{(w)}=2 \tau_{a}^{(w)}=2, \\
& \beta_{t}^{(w)}=z \tau_{t}^{(w)}=5 / 4,
\end{aligned}
$$




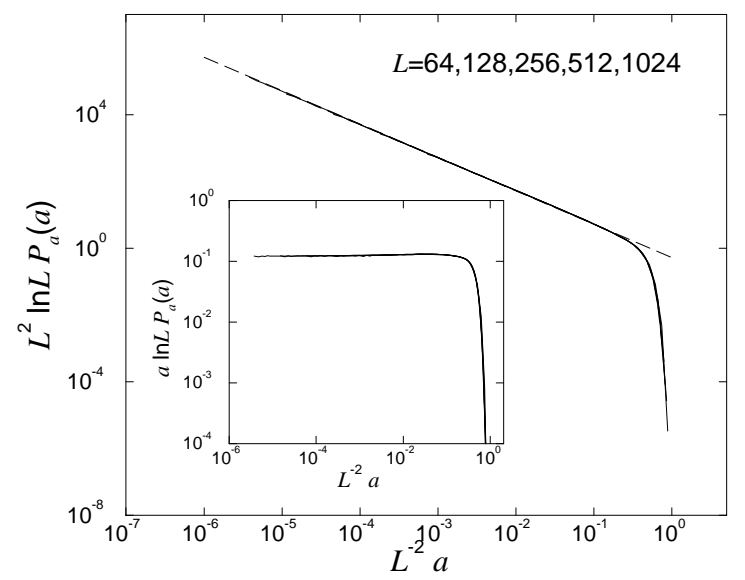

FIG. 4. Finite-size scaling plot of the wave area distribution $P_{a}^{(w)}(a)$ for bulk waves in $D=2$. The perfect data collapse shows that $d_{f}=2$ and $\beta_{a}^{(w)}=2$, as predicted analytically. The dashed line represents a power law with exponent $\tau_{a}^{(w)}=1$. The factor $\ln L$ comes from the normalization of the distributions. The inset verifies the scaling ansatz Eq. (更).

for bulk waves and

$$
\begin{aligned}
& \beta_{a}^{\text {(boundary })}=2 \tau_{a}^{\text {(boundary })}=3, \\
& \beta_{t}^{\text {(boundary })}=z \tau_{t}^{\text {(boundary })}=9 / 4,
\end{aligned}
$$

for boundary waves.

The finite-size scaling plot for the area distribution $P_{a}^{(w)}(a)$ of bulk waves in $D=2$ is shown on Fig. 团. In the inset of this Figure, as well as in the insets of plots for other distributions of waves, we show the collapses according to ansatz of Eq. (1). Taking $\beta_{a}^{(w)}=2$ and $d_{f}=2$ we see a perfect data collapse. The finite-size scaling ansatz of the duration distribution is plotted in Fig. 5 .

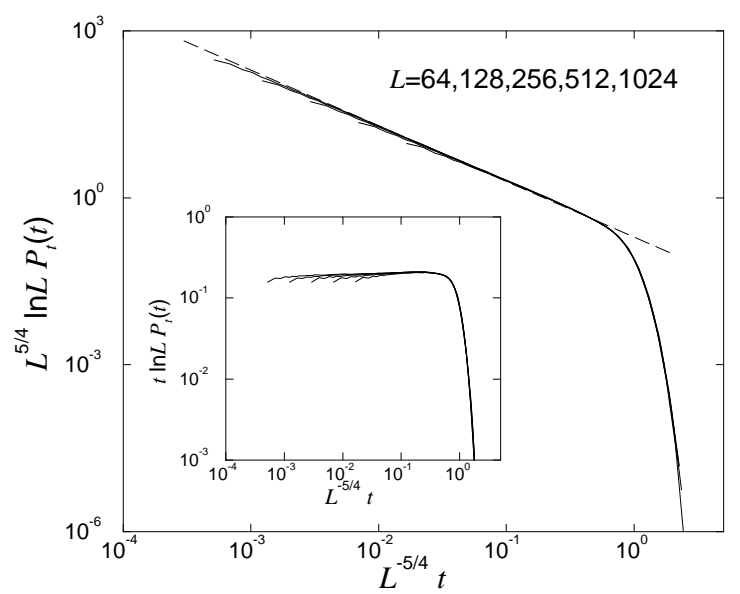

FIG. 5. Finite-size scaling plot of the wave duration distribution $P_{t}^{(w)}(t)$ for bulk waves in $D=2$. Here, the data collapse is achieved with $\beta_{t}^{(w)}=\nu_{t}^{(w)}=5 / 4$ which confirms again Eq. (31). The dashed line represents a power law with exponent $\tau_{t}^{(w)}=1$. Again, the normalization factor $\ln L$ is needed for a good data collapse.

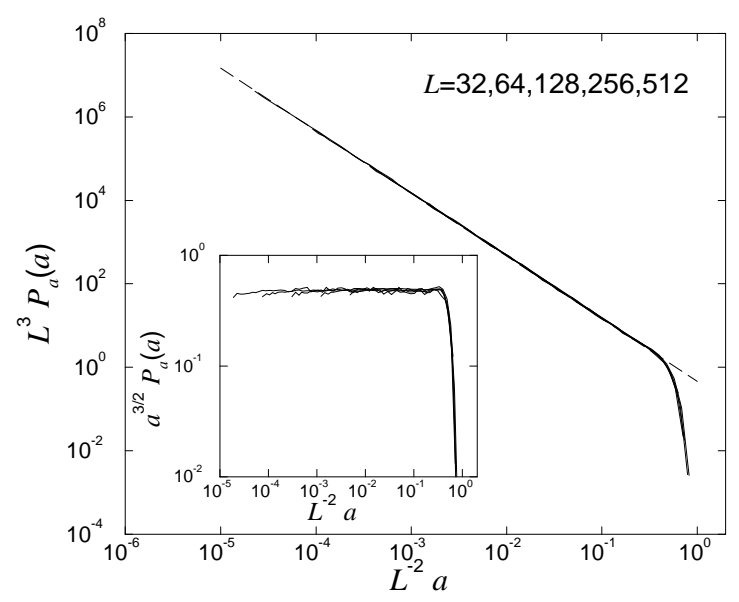

FIG. 6. Same as Fig. 4 , but for boundary waves in $D=2$. Again the data collapse is obtained with the predicted values $\beta_{a}^{\text {(boundary) }}=3$ and $\mathrm{d}_{f}=2$. The dashed line corresponds to $\tau_{a}^{\text {(boundary) }}=3 / 2$, as predicted theoretically.

These data confirm that waves are not fractal, and that their duration is as predicted by the correspondence with spanning trees and loop-erased random walks.

As was mentioned above, the avalanches started at the boundary consist of a single wave, so for this type of avalanches the distribution of avalanches coincides with that of waves. The finite-size scaling plots for the area and duration distributions for boundary waves are shown in Fig. 6 and Fig. 7, respectively. Again all predictions are verified, in particular we see that $d_{f}=2$, i.e. also boundary waves are not fractal in $D=2$.

Finally, let us consider avalanches starting at a finite distance from the boundary. The crossover from the boundary to bulk behavior of the wave distribution should be described by Eq. (20). More precisely, the equipotential surfaces in $D=2$ are circles [28] with ra-

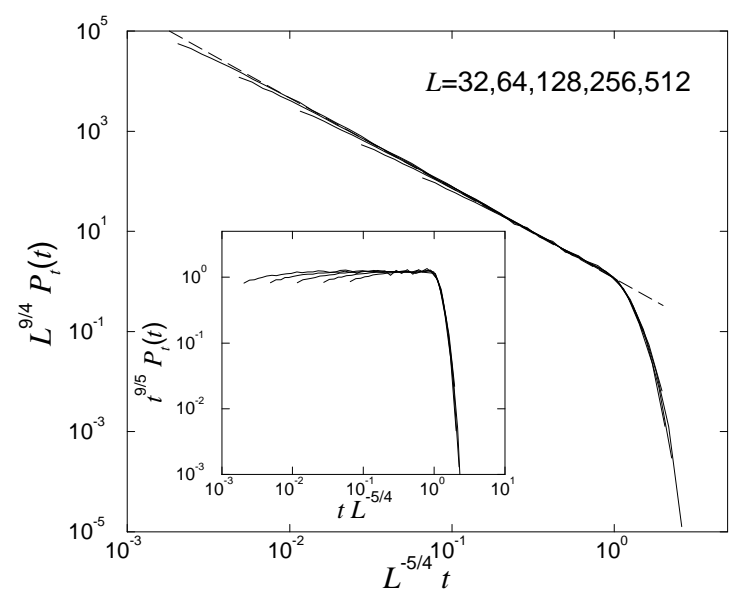

FIG. 7. Same as Fig. 月, but for boundary waves in $D=2$. Again the data collapse is obtained with the predicted values $\beta_{t}^{\text {(boundary) }}=9 / 4$ and $\nu_{t}^{(w)}=5 / 4$. The dashed line corresponds to $\tau_{t}^{\text {(boundary) }}=9 / 5$, as predicted theoretically. 


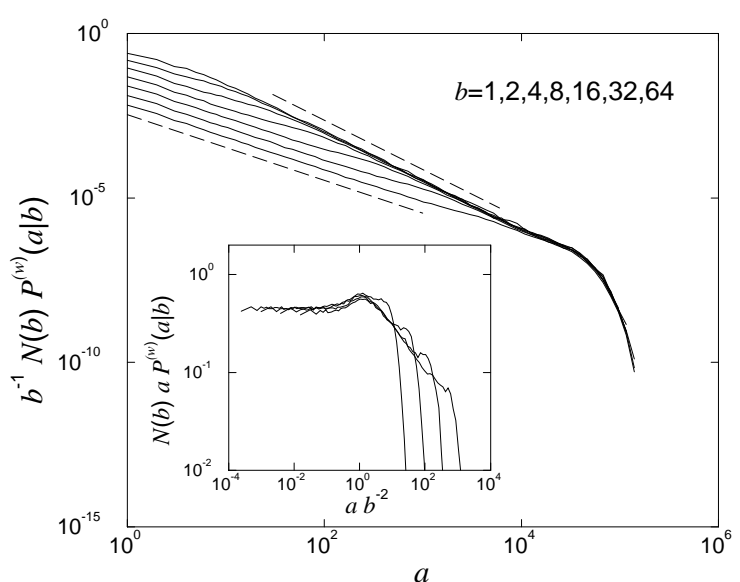

FIG. 8. Area distributions of waves initiated at different distances $b$ from the boundary for a system of size $256 \times 1024$ with cylindrical boundary conditions. Each curve is averaged over approximately $10^{6}$ avalanches. The dashed lines correspond to the bulk $\left(\tau_{a}^{(w)}=1\right)$ and boundary $\left(\tau_{a}^{\text {(boundary) }}=3 / 2\right)$ scaling behavior, respectively. The distributions are multiplied by $b^{-1} N(b), N(b)=\int P_{a}^{(w)}(a \mid b) d a$ in order to have the curves collapsing for large $a$. Inset: the rescaled distributions for $b=8,16,32,64$ demonstrate that the crossover from bulk to boundary behavior takes place at values of area of order $b^{2}$.

dius $\xi$ and $G \sim \ln \left[\left(b^{2} / \xi^{2}+1\right)^{1 / 2}-b / \xi\right]$. In the scaling region where $\rho(\xi)=1$, we have therefore $P_{a}^{(w)}(a \mid b)=$ $-(d a / d \xi)^{-1} d G / d \xi$ which gives

$$
P_{a}^{(w)}(a \mid b) \sim \frac{b}{a \sqrt{a+\pi b^{2}}} \sim \begin{cases}b / a^{3 / 2} & \text { for } a>\pi b^{2} \\ 1 / a & \text { for } a<\pi b^{2}\end{cases}
$$

To check this, we simulated the BTW model with cylindrical boundary conditions, and collected data for waves started at distance $b$ from the open boundary. The results are plotted in Fig. 8. For small $a$ we see indeed the bulk behavior which crosses over to the boundary behavior $a^{-3 / 2}$ for $b^{2}<a<L^{2}$. In the latter region we also see clearly the linear dependence on $b$.

The above shows that our understanding of waves in the two-dimensional BTW model is basically complete. In contrast, and in spite of numerous efforts, the scaling behavior of avalanches in the two-dimensional BTW model is still an open problem. This is due to multiple topplings. The average number of waves in an avalanche scales as [2]

$$
\langle n\rangle \sim \ln L .
$$

There are also several results known about correlations in the sizes of successive waves $[9,21,37,39$. . Nevertheless, even most basic questions such as the distribution of $n$ or the dependence of $n$ on the area $a$ are not yet solved.

Equation (34) would be most easily explained if $P_{n}(n)$ were simply $\sim 1 / n^{2}$. Present data seem to agree with this for the largest lattices (Fig. 9), but actually the data are better fited with a power $1 / n^{2.1}$ than with $1 / n^{2}$ (see

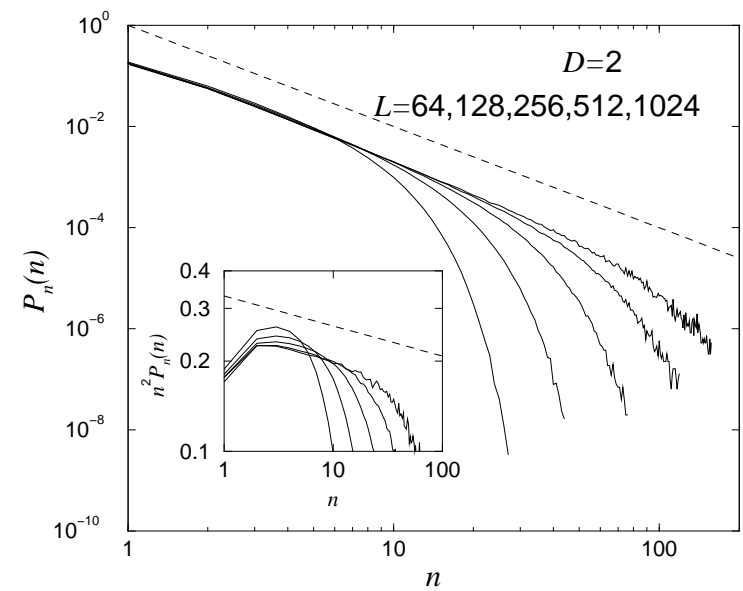

FIG. 9. Probability distribution of the number of waves $n$ in an avalanche for $D=2$. The dashed line corresponds to the power-law behavior $P_{n}(n) \sim n^{-2}$ as predicted in 15. The data were collected from avalanches initiated at the center region of the square lattice with cylindrical boundaries. The inset shows the same data multiplied by $n^{2}$ There, the dashed line is $\propto n^{-0.1}$.

inset). Similar results are obtained for $\langle n\rangle_{a}$, the average number of waves in avalanches with fixed $a$ (Fig. 10). Although they seem to scale like a power of $a$, as assumed in 15], a closer study shows significant deviations which seem hard to explain as finite size effects.

There are several recent papers 10,40 which try to explain these problems by unexpected features of avalanches which reach the boundary. But data such as those shown in Figs. 1, 10 indicate that there are already problems with avalanches which do not reach the boundary.

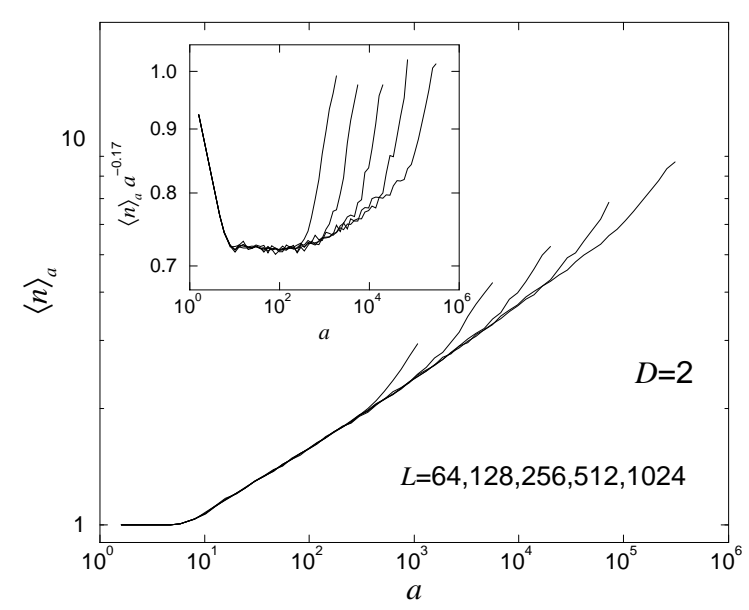

FIG. 10. Average number of waves $\langle n\rangle_{a}$ as a function of the avalanche area $a$ for $D=3$ and various system sizes $L$. Although the main figure looks straight at first, the inset displaying the rescaled average shows significant deviations from the assumed pure power-law behavior 15. The data were collected from non-dissipative avalanches initiated at the center region of the square lattice with open boundaries. Thus the curvature seen in the inset cannot come from avalanches reaching the boundary. 


\section{B. $\mathrm{D}=3$}

Simulations of waves of the BTW model in $D=3$ also give good agreement with our analytical results of Sections IV and V. For bulk waves we now have $\beta_{a}^{(w)}=4, \quad \tau_{a}^{(w)}=4 / 3, \beta_{t}^{(w)}=1 / \nu_{\text {LERW }}+1 \approx 2.623$, and $\tau_{t}^{(w)}=1+\nu_{\text {LERW }} \approx 1.616$. For boundary waves, the corresponding numbers are $\beta_{a}^{\text {(boundary) }}=5, \tau_{a}^{\text {(boundary) }}=$ $5 / 3, \beta_{t}^{\text {(boundary) }}=1 / \nu_{\text {LERW }}+2 \approx 3.623$, and $\tau_{t}^{\text {(boundary) }}=$ $1+2 \nu_{\text {LERW }} \approx 2.232$. For these $\beta$ values, the data collapses of bulk (Fig. 11,12) and boundary (Fig. 13, 14) waves are perfect. They confirm also the analytical predictions for the $\tau$ exponents, verifying in particular that the waves have fractal dimension $d_{f}=3$.

Due to the rarity of multiple topplings, avalanche distributions coincide within the displayed accuracy with wave distributions, when plotted as in Fig. 11 and Fig. 12. In order to show significant results for multiple topplings, we have to present the data differently. In Fig. 15 we plotted the average number of waves at fixed $a,\langle n\rangle_{a}$, against $\log a$. Neither using a logarithmic scale for $\langle n\rangle_{a}$ (main figure) nor a linear scale (inset) give perfectly straight lines. Thus the data can be interpreted either as a power law with a very small exponent,

$$
\langle n\rangle_{a} \sim a^{\alpha}, \quad \alpha \approx 0.06
$$

or as a logarithmic growth.

In the latter case, we would of course have $\tau_{a}^{(w)}=\tau_{a}=$ $\tau_{s}$. In the opposite case of a power law with exponent $\alpha \approx 0.06$ we can give crude estimates for the differences between these $\tau$ exponents, using some heuristic assumptions.

The first assumption is that different waves in the same avalanche involve essentially the same sites. If this is true, we should have $P_{a}^{(w)}(a) d a \approx\langle n\rangle_{a} P_{a}(a) d a$. Using this together with Eq. (35), we find $\tau_{a}=4 / 3+\alpha \approx$

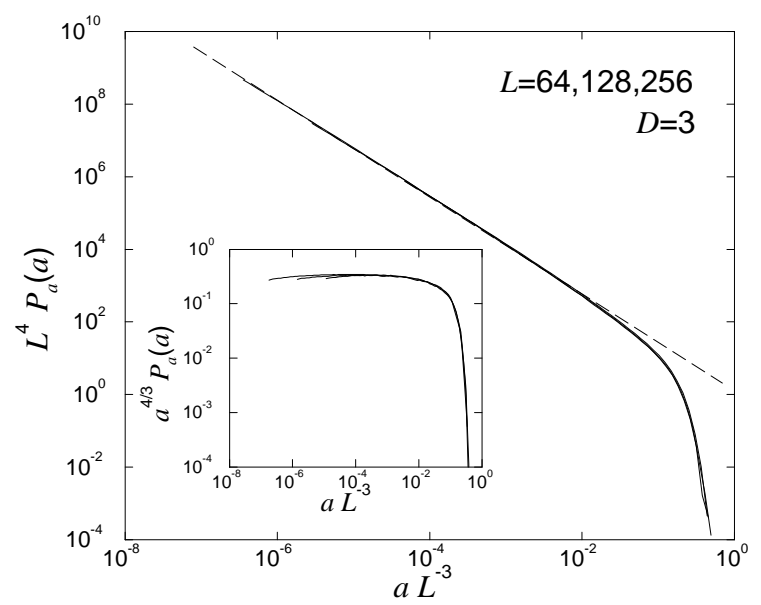

FIG. 11. Finite-size scaling plots of the area distribution $P_{a}^{(w)}(a)$ for bulk avalanches in $D=3$. Assuming compact avalanches $\left(d_{f}=3\right)$ we get good data collapses and the resulting $\tau_{a}^{(w)}$ exponent agrees with the theoretical prediction (dashed lines).

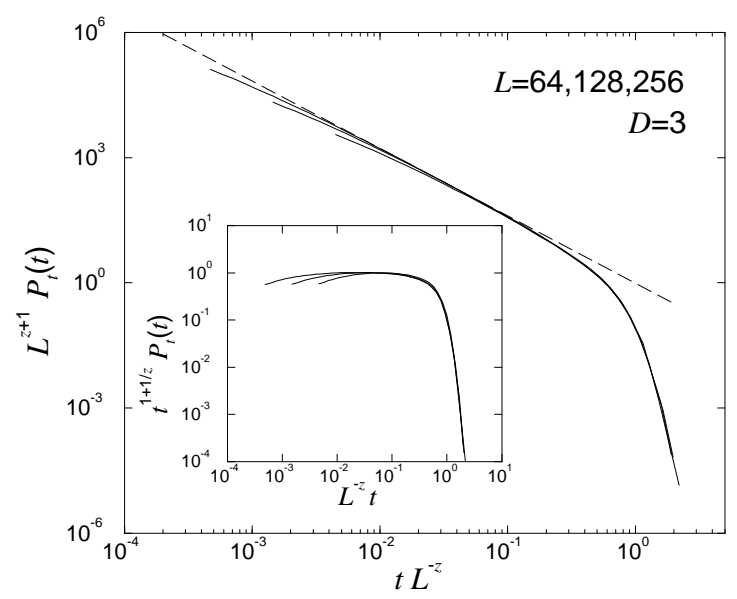

FIG. 12. Finite-size scaling plots of the duration distribution $P_{t}^{(w)}(t)$ for bulk avalanches in $D=3$. Using compact avalanches $\left(z=1 / \nu_{\text {LERW }}\right)$ we get good data collapses and the resulting $\tau_{t}^{(w)}$ exponent agrees with the theoretical prediction (dashed lines).

1.39. Since the basic assumption here is most likely not justified, this is only a very crude (and most likely too large, in particular since the growth of $\langle n\rangle_{a} P_{a}(a) d a$ could be logarithmic) estimate for the difference between $\tau_{a}$ and $\tau_{a}^{(w)}$.

An estimate for the difference between $\tau_{a}$ and $\tau_{s}$ is obtained as follows. An upper bound for the size of an avalanche of area $a$ containing $n$ waves is $s<n a$. Therefore, the assumption $s \sim\langle n\rangle_{a} a$ leads to the maximal difference between the area and size exponents. Using Eq. (35) we get $a \gtrsim s^{1 /(1+\alpha)}$. Then, Eq. (7) gives $\left(\tau_{s}-1\right) \geq\left(\tau_{a}-1\right) /(1+\alpha)$ and we can conclude that

$$
\tau_{a}-\tau_{s} \leq \frac{\alpha}{1+\alpha}\left(\tau_{a}-1\right) \leq 0.02
$$

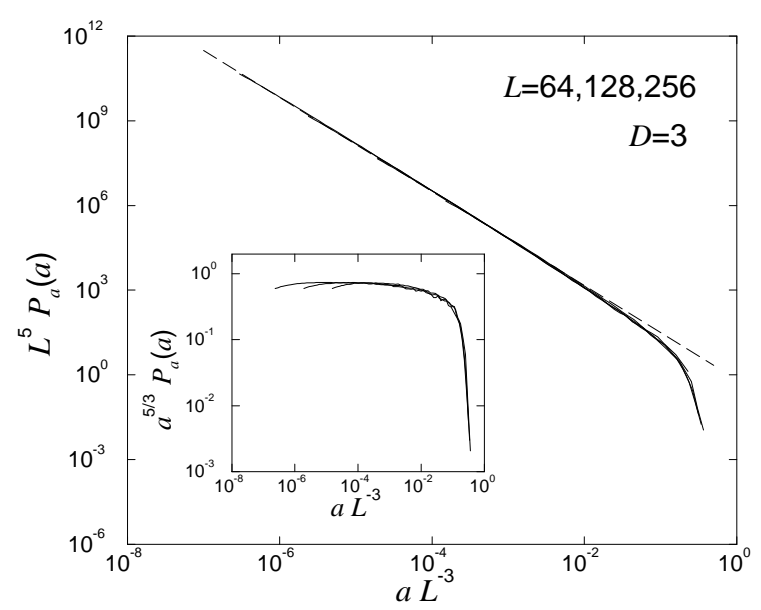

FIG. 13. Finite-size scaling plots of the area distribution for boundary waves in $D=3$. The data collapses confirm again $d_{f}=3$, and the dashed line demonstrates the agreement with the predicted value for $\tau_{a}^{\text {(boundary) }}$. 


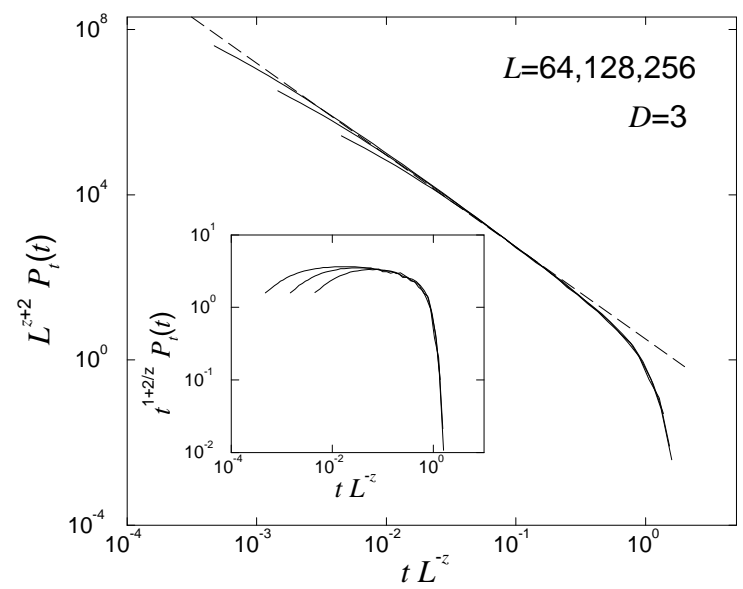

FIG. 14. Finite-size scaling plot of the duration distributions for boundary waves in $D=3$. The data collapses confirm again $z=1 / \nu_{\text {LERW }}$, and the dashed line demonstrates the agreement with the predicted value for $\tau_{t}^{\text {(boundary) }}$.

A direct verification of such slight differences between the $\tau$ exponents could be tried by plotting ratios of the distributions, as was done in Fig. 1 for $D=2$. We do not show any such ratio here, since they are all very close to 1 in the region where the distributions should follow power laws, and the deviations from 1 seem not to be simple powers.

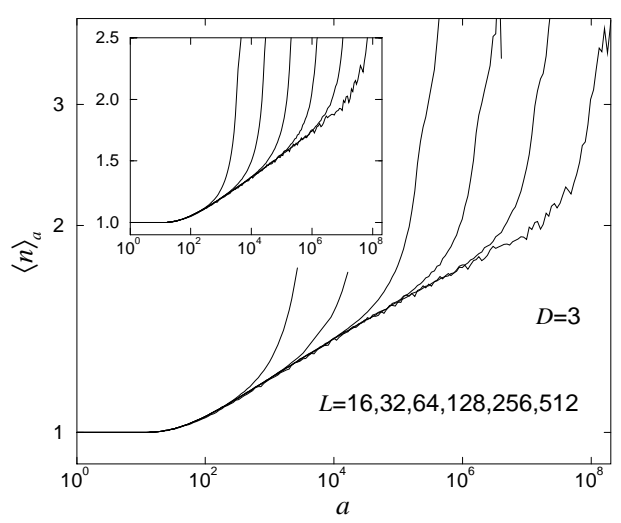

FIG. 15. Average number of waves $\langle n\rangle_{a}$ as a function of the avalanche area $a$ for $D=3$ and various values of the system size $L$. In order to minimize finite size effects, cylindrical boundary conditions with one open and two periodic directions were used and one half of all sand grains were thrown onto the central planes $y=L / 2$ and $y=L / 2+1$. The $\mathrm{y}$-axis is logarithmic in the main plot, and linear in the inset. Neither way of plotting gives perfectly straight lines in the region where that data for different $L$ collapse. Although the main figure looks more straight at first sight, a more careful inspection shows a slight downward curvature.

\section{C. $\mathrm{D}=4$}

As the dimension of the BTW model increases, multiple toppling events in avalanches become more and more rare. For $D \geq 4$ it was shown in [26] that $\langle n\rangle_{a}$ grows not faster than logarithmically, i.e. $\alpha=0$. As we already mentioned in the previous subsection, this means $\tau_{a}^{(w)}=\tau_{a}=\tau_{s}$, i.e., the scaling behavior of waves and avalanches in $D=4$ is characterized by the same exponents and scaling functions.

At the upper critical dimension $D_{\mathrm{u}}=4$ logarithmic corrections to the simple scaling are essential. The probability distributions of the radius, duration, area and the scaling relations between them are expected to have the form (cf. [25])

$$
P_{r}(r) \sim \frac{(\ln r)^{x_{r}}}{r^{3}}, P_{t}(t) \sim \frac{(\ln t)^{x_{t}}}{t^{2}}, P_{a}(a) \sim \frac{(\ln a)^{x_{a}}}{a^{3 / 2}},
$$

and

$$
a \sim \frac{r^{4}}{(\ln r)^{N_{a}}}, \quad t \sim \frac{r^{2}}{(\ln r)^{N_{t}}},
$$

respectively.

The exponents of logarithmic corrections $x_{r}, x_{a}, x_{t}$, $N_{a}$, and $N_{t}$ obey the scaling relations [25]

$$
x_{r}=x_{a}+N_{a} / 2, \quad x_{r}=x_{t}+N_{t}
$$

which follow straightforwardly from Eqs. (37) and (38).

Using arguments similar to those at the end of the previous subsection, we obtain an inequality for the exponents of logarithmic corrections for waves and avalanches

$$
x_{a}^{(w)}=x_{a} \leq x_{s} .
$$

This allows us to compare below analytical estimations for waves with numerical results for avalanches.

It follows from Lawler's results [34 discussed in Sect. V that $N_{t}=1 / 3$ exactly. But as with all logarithmic corrections, a numerical verification is not easy. The main reason is that the logarithms are never very much larger than 1, even for the largest simulations. Therefore the next-to-leading terms (which are typically suppressed by powers of the same logarithms) are in general not negligible. In view of this, the disagreement with recent simulations [25] which had suggested $N_{t} \approx 1 / 2$ should not be taken serious. Data for the mean squared radius of avalanches with fixed duration $t$ are shown in Fig. 16 . More precisely, since we expect

$$
\frac{\langle r\rangle_{t}^{2}}{t} \sim(\ln t)^{1 / 3}
$$

we plotted $\left[\langle r\rangle_{t}^{2} / t\right]^{3}$ against $\ln t$. Apart from very large $t$ when the finiteness of the lattice makes itself seen, we observe essentially a straight line (which is a bit fortuitious since there are also $1 / t$ corrections which are important for small $t$ ). At the same time, a power law dependence $\langle r\rangle_{t}^{2} \sim t^{2 \nu}$ with $\nu>1 / 2$, as would be expected if $D_{c}>4$, seems ruled out. 


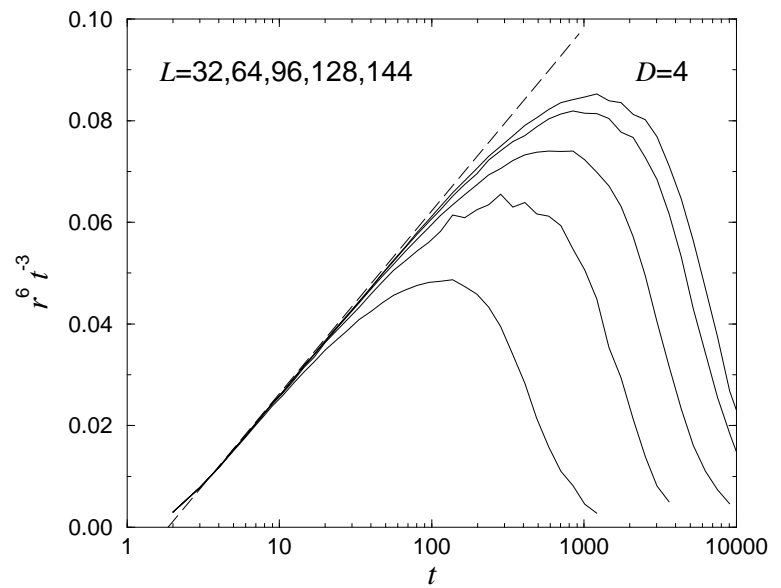

FIG. 16. The gyration radius of avalanches as a function of their duration. We plot the sixth power of the average rescaled gyration radius, $\left[r_{g}^{2} t^{-1}\right]^{3}$, in a logarithmic diagram, since this should result in a straight line according to Eq. (41). Such a linear regime is indeed observed (dashed line; its slope and intercept are not predicted by theory), and it increases with the system size $L$.

For the other exponents $x_{r}, x_{a}, x_{t}, N_{a}$ we can only give inequalities from the analytical results of section $\mathrm{V}$. The upper bound Eq. (27) for the density of waves leads to the relation

$$
\rho(r) \sim(\ln r)^{-\delta}, \quad \delta \geq 1 .
$$

Using this asymptotics and Eq. (11) we get

$$
P_{r}^{(w)}(r) \sim G(r) / \rho(r) \sim \frac{(\ln r)^{\delta}}{r^{3}},
$$

which gives $x_{r}=\delta$. The area $a$ of a wave scales in leading order as

$$
a \sim \int_{1}^{r} \rho\left(r^{\prime}\right) r^{\prime 3} d r^{\prime} \sim \frac{r^{4}}{(\ln r)^{\delta}}
$$

giving $N_{a}=\delta$. Hence, from Eq. (39) we have

$$
x_{a}=\delta / 2 \geq 1 / 2 .
$$

In order to verify these predictions - and to verify, in the first place, that deviations from power laws with the mean field exponents $\tau_{r}=3, \tau_{t}=2, \tau_{a}=3 / 2$ cannot be eliminated by changing these exponents - we performed extensive simulations. Numerical data of the size distribution $P_{s}(s)$ are shown in Fig. 17. Lattice sizes range from $L=32$ to $L=144$. After multiplying with $s^{3 / 2}$, we see indeed no indication that the remaining $s$ dependence follows a clean power law. In order to find the expected logarithmic corrections, we multiplied these data by $(\ln s)^{x_{a}}$, with several trial values for the exponent $x_{a}$. Taken at face value, this would give best fits with $x_{a} \approx 0.25$. In view of inequality (39) and of the difficulties in obtaining correct logarithmic corrections mentioned above, we propose that indeed $x_{a}=1 / 2$. From the relations Eq. (39) we get then $x_{r}=N_{a}=1$ and $x_{t}=2 / 3$.

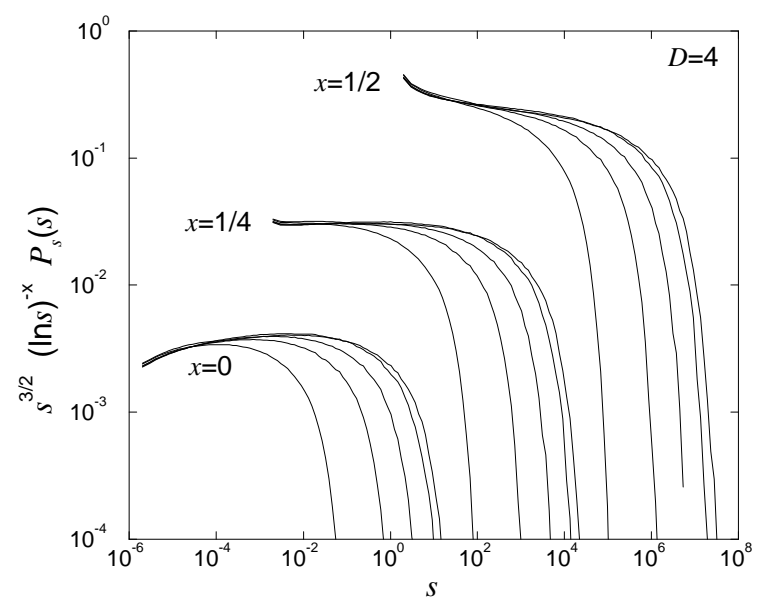

FIG. 17. Area distributions for avalanches in $D=4$. In order to render the plots more significant, first of all the dominant $s$-dependence was removed by multiplying with $s^{3 / 2}$. Then, in order to check whether the remaining $s$-dependence in the scaling region is compatible with logarithmic corrections as proposed in Eq. (37), we also divided by powers $(\ln s)^{x}$ and shifted the resulting curves horizontally and vertically in order to avoid overlaps. The best agreement is found with $x \approx 0.25$.

\section{CONCLUSIONS}

We have studied probability distributions of waves of topplings in the BTW model on $D$-dimensional rectangular lattices for $D \geq 2$. We have proved analytically that waves as well as boundary avalanches do exhibit critical behavior and that their probability distributions display power law asymptotics. We have derived exact values of critical exponents of these distributions. We have proven analytically that the upper critical dimension of the BTW model is $D_{\mathrm{u}}=4$, showing that previously observed deviations from mean field behavior at $D=4$ [17,38] are due to logarithmic corrections. All these results have been confirmed by extensive numerical simulations. During these simulations we have also verified that wave distributions follow the standard finite size scaling ansatz. The exponent of the leading logarithmic correction to the distribution of avalanche life times (or, more precisely, lifetimes of waves) has been derived exactly from known asymptotics of loop-erased random walks. Estimations are given for the exponents of the logarithmic corrections to the other distributions.

We therefore have now a rather complete picture of the dynamics of single waves in the BTW model for all dimensions. For $D \geq 4$ this means that we also understand avalanche dynamics, since multiple topplings are so rare that they can be neglected. For $D=2$ the latter is certainly not true, and our understanding of avalanche dynamics is still incomplete. For $D=3$, finally, multiple topplings represent a small but not negligible effect, and we have hope that the problem will be solved soon. 


\section{ACKNOWLEDGMENTS}

The authors thank E. V. Ivashkevich for very helpful remarks. DVK acknowledges the financial support from the Alexander von Humboldt Foundation and the kind hospitality of the Computational Physics group in Duisburg University, where part of this work was done. VBP was supported by RFBR through Grant No 99-01-00882 and by the Heisenberg-Landau program. He also thanks NIC, Forschungszentrum Jülich for the support and hospitality.

[1] P. Bak, C. Tang, and K. Wiesenfeld, Phys. Rev. Lett. 59, 381 (1987).

[2] D. Dhar, Phys. Rev. Lett. 64, 1613 (1990).

[3] V. B. Priezzhev, J. Stat. Phys. 74, 955 (1994).

[4] E. V. Ivashkevich, J. Phys. A 27, 3643 (1994).

[5] S.P. Obukhov, in Random Fluctuations and Pattern Growth, edited by H. E. Stanley and N. Ostrowsky, NATO ASI Series E: Applied Sciences Vol. 157 (Kluwer, Dordrecht, 1988).

[6] Y.-C. Zhang, Phys. Rev. Lett. 63, 470 (1989).

[7] L. Pietronero, A. Vespignami, and S. Zapperi, Phys. Rev. Lett. 72, 1690 (1994).

[8] E. V. Ivashkevich, Phys. Rev. Lett. 76, 3368 (1996).

[9] V. B. Priezzhev, D. V. Ktitarev, and E. V. Ivashkevich, Phys. Rev. Lett. 76, 2093 (1994).

[10] M. De Menech, A. L. Stella, and C. Tebaldi, Phys. Rev. E 58, 2677 (1998).

[11] C. Tebaldi, M. De Menech, and A.L. Stella, preprint cond-mat/9903270 (1999).

[12] A. Chessa, H. E. Stanley, A. Vespignani, and S. Zapperi, preprint cond-mat/980263 (1998).

[13] S. Lübeck and K.D. Usadel, Phys. Rev. E 55, 4095 (1997)

[14] A. A. Ali and D. Dhar, Phys. Rev. E 52, 4804 (1995).

[15] S. N. Majumdar and D. Dhar, Physica A 185, 129 (1992).

[16] S.S. Manna, Physica (Amsterdam) 179A, 249 (1991).

[17] K. Christensen and Z. Olami, Phys. Rev. E 48, 3361 (1993).

[18] E. Milshtein, O. Biham, and S. Solomon, Phys. Rev. E 58, 303 (1998).

[19] E. V. Ivashkevich, D. V. Ktitarev, and V. B. Priezzhev, Physica A 209, 347 (1994).

[20] P. Grassberger and S. S. Manna, J. Phys. (France) 51, 1077 (1990).

[21] M. Paczuski and S. Boettcher, Phys. Rev. E 56, R3745 (1997).

[22] D. Dhar and S. S. Manna, Phys. Rev. E 49, 2684 (1994).

[23] E. V. Ivashkevich, D. V. Ktitarev, and V.B. Priezzhev, J. Phys. A 27, L585 (1994).

[24] A. L. Stella, C. Tebaldi, and G. Caldarelli, Phys. Rev. E 52, 72 (1995).

[25] S. Lübeck, Phys. Rev. E 58, 2957 (1998).

[26] V. B. Priezzhev, preprint cond-mat/9904054 (1999).

[27] S. N. Majumdar and D. Dhar, J. Phys. A 24, L357 (1991).
[28] J. D. Jackson, Classical electrodynamics (Wiley, New York, 1975).

[29] S. N. Majumdar, Phys. Rev. Lett. 68, 2329 (1992).

[30] G. F. Lawler, Duke Math. J. 47, 655 (1980).

[31] A. Coniglio, Phys. Rev. Lett. 62, 3054 (1989).

[32] A. J. Guttmann and R. J. Bursill, J. Stat. Phys. 59, 1 (1990).

[33] R. E. Bradley and S. Windwer, Phys. Rev. E 51, 241 (1995).

[34] G. F. Lawler, Intersection of Random Walks (Birkhäuser, Boston, 1991).

[35] L. P. Kadanoff, S. R. Nagel, L. Wu, and S. M. Zhou, Phys. Rev. A 39, 6524 (1989).

[36] S. Lübeck and K.D. Usadel, Phys. Rev. E 56, 5138 (1997).

[37] D. V. Ktitarev and V.B. Priezzhev, Phys. Rev. E 58, 2883 (1998).

[38] A. Chessa, E. Marinari, A. Vespignani, and S. Zapperi, Phys. Rev. E 57, 6241 (1998).

[39] E. V. Ivashkevich, V.B. Priezzhev, C. K. Hu, and C. Y. Lin, to be published (1999).

[40] B. Drossel, preprint cond-mat/9904075 v2 (1999). 